VoL. 52 (1995) [235-246]

\title{
SOME UTILITY THEOREMS ON INDUCTIVE LIMITS OF PREORDERED TOPOLOGICAL SPACES
}

\author{
J.C. Candeal, E. Induráin and G.B. Mehta
}

We prove the existence of an order-preserving function on a class of preordered topological spaces that are inductive limits of preordered spaces. Some applications to economics are given.

\section{INTRODUCTION}

One of the main problems in utility theory is to find conditions which imply the numerical representability of topological spaces on which a preorder, or partial order, is defined. This study has reached a wide development in the literature: see, for instance, the works of Eilenberg, Debreu, Fleischer and Jaffray on completely preordered topological spaces $[9,7,10,15]$, and the seminal book by Nachbin together with the papers by Mehta and Herden $[\mathbf{2 2}, \mathbf{2 0}, \mathbf{2 1}, \mathbf{1 1}, \mathbf{1 2}]$, in the framework of preordered topological spaces.

In general, the existence of a continuous representation is closely related to topological conditions that lie "in the neighbourhood of separability" (see [4] and [5]).

Recently, some very general conditions for the existence of a continuous order preserving function have been obtained by Herden [11, 12]. The work of Herden is based on Nachbin's theory of normally preordered spaces.

The objective of this paper is to consider general families of preordered (or partially ordered) spaces that admit a representation in a natural way. The idea of using a family of preference relations on a space has been outlined by Aumann [3, p.241] and others.

In this paper we introduce another class of preordered spaces which admit a (utility) representation, the class of topological spaces that are inductive limits of preordered spaces. In particular, our main result proves the existence of a continuous representation of a topological space that is a suitable inductive limit of compact order-separable spaces. Then we apply this result to locally compact $\sigma$-compact preordered spaces and completely preordered topological vector spaces. Also, we extend the result to a broader class of spaces showing through an example that some elements in this class are neither compact nor order-separable. We conclude by using the idea of an inductive limit to make precise the notion of a local utility function and to prove an infinite-dimensional version of the classical Arrow-Hahn theorem on utility functions.

Received 14 December 1994

Copyright Clearance Centre, Inc. Serial-fee code: 0004-9729/95 \$A2.00+0.00. 


\section{Preliminaries}

A preorder $\leqslant$ on a topological space $X$ is a reflexive transitive binary relation on $X$. We shall denote $x<y$ if and only if $x \leqslant y$ and not $y \leqslant x$, and $x \sim y$ when $x \leqslant y$ and $y \leqslant x$.

The preorder $\leqslant$ is said to be:

(i) complete or decisive if for two elements $x, y \in X$, either $x \leqslant y$ or $y \leqslant x$,

(ii) an order if it is an antisymmetric binary relation,

(iii) a total order (or chain), if it is a complete (or decisive) order,

(iv) closed if its graph in the square $X^{2}$ is a closed subset of the topological space $X^{2}$,

(v) continuous if the sets $\{x \in X ; x \leqslant y\}$ and $\{x \in X ; y \leqslant x\}$ are closed for every $y \in X$,

(vi) representable if there exists an increasing and order-preserving map $u: X \rightarrow$ $\mathbb{R}$, that is, $x \leqslant y$ implies $u(x) \leqslant u(y)$ and $x<y$ implies $u(x)<u(y)$. The function $u$ is called a utility function,

(vii) continuously representable if there exists a continuous utility function which represents $\leqslant$. ( $\mathbb{R}$ is endowed with the usual topology).

For $T \subset X$ we denote by $\mathrm{cl}(T)$ the closure of $T$. The preorder $\leqslant$ is said to be spacious if $x<y$ implies $\operatorname{cl}(\{z \in X ; z<x\}) \subset\{z \in X ; z<y\}$.

A subset $E \subset X$ is said to be decreasing if $b \in E, a \leqslant b$ imply that $a \in E$. Similarly, one defines the concept of an increasing set. A topological space equipped with a preorder is said to be normally preordered, if, for every two disjoint closed subsets $A, B \subset X, A$ being decreasing and $B$ increasing, there exists two disjoint open subsets $A^{*}$ and $B^{*}$ such that $A^{*}$ contains $A$ and is decreasing, and $B^{*}$ contains $B$ and is increasing.

A preordered topological space $(X, \leqslant)$ is said to be order-separable if there exists a countable subset $Z \subset X$ such that if $x, y \in X$ and $x<y$, then there exists a $z \in Z$ such that $x<z<y$.

\section{REPRESENTABILITY OF INDUCTIVE LIMITS OF COMPACT PREORDERED SPACES}

In this section we obtain some existence results of utility functions on topological preordered spaces which are inductive limits of compact order-separable preordered spaces.

Definition: A compact preordered space $X$ is a compact space equipped with a closed preorder.

Lemma 1. Let $X$ be a compact preordered space. Then the quotient space $X / \sim$ is a compact ordered space in the sense of Nachbin (that is, a compact space endowed 
with a closed order).

Proof: $X / \sim$ is a compact space since the projection map $p: X \rightarrow X / \sim$ is continuous. Moreover, it is plain that $\leqslant$ induces an order $\leqslant$ on $X / \sim$ in the natural way. Let us show that $\leqslant$ is closed. To this end, let $\left(\left[a_{\alpha}\right],\left[b_{\alpha}\right]\right)$ be a net, belonging to the graph of the relation $\leqslant^{\prime}$, and converging to $([a],[b]) \in(X / \sim)^{2}$. Because $\left[a_{\alpha}\right] \leqslant \leqslant^{\prime}\left[b_{\alpha}\right]$, it follows that $a_{\alpha} \leqslant b_{\alpha}$. So $\left(a_{\alpha}, b_{\alpha}\right)$ belongs to the graph of the relation $\leqslant$. Now notice that the graph of $\leqslant$ is actually a compact subset of $X^{2}$. Hence there exists a subnet $\left(a_{\beta}, b_{\beta}\right)$ converging to a point $(x, y)$ in the graph of $\leqslant$, that is, $x \leqslant y$. Thus $x \in[a]$, $y \in[b]$ and therefore $[a] \leqslant[b]$, that is, $\leqslant^{\prime}$ is closed.

Now, we combine some results due to Nachbin, Mehta, and Herden to obtain an existence theorem for order-preserving functions defined on inductive limits of compact preordered spaces.

First, we recall the definition of directed sets and inductive limits:

DEFINITION: A directed set $(D, \leqslant)$ is a preordered set with the following property: For each $a, b \in D$ there exists an element $c \in D$ such that $a \leqslant c$ and $b \leqslant c$.

A subset $E \subset D$ is said to be cofinal in $D$, if for every $d \in D$ there exists $e \in E$ such that $d \leqslant e$.

Let $D$ be a directed set and let $\left\{X_{\alpha} ; \alpha \in D\right\}$ be a family of topological spaces, indexed by $D$. For each pair of indices $\alpha, \beta$ satisfying $\alpha \leqslant \beta$ assume that there is given a continuous map $\zeta_{\alpha, \beta}: X_{\alpha} \rightarrow X_{\beta}$, and that these satisfy the conditions: (i) $\zeta_{\alpha, \alpha}$ is the identity on $X_{\alpha}$; (ii) if $\alpha \leqslant \beta \leqslant \gamma$, then $\zeta_{\alpha, \gamma}=\zeta_{\beta, \gamma} \circ \zeta_{\alpha, \beta}$.

Then this family $\left\{X_{\alpha}, \zeta_{\alpha, \beta}\right\}$ of spaces and maps is called an inductive spectrum over $D$, with spaces $X_{\alpha}$ and connecting maps $\zeta_{\alpha, \beta}$.

The image of an element $x_{\alpha} \in X_{\alpha}$ under any connecting map is termed a sucessor of $x_{\alpha}$. Each inductive spectrum $\left\{X_{\alpha}, \zeta_{\alpha, \beta}\right\}$ yields a limit space in the following way: Let $S=\sum\left\{X_{\alpha} ; \alpha \in D\right\}$ be the free union of the spaces, endowed with the final topology relative to the inclusions $\iota_{\alpha}: X_{\alpha} \rightarrow S$. Two elements $x_{\alpha} \in X_{\alpha}, x_{\beta} \in X_{\beta}$ in $S$ are said to be equivalent whenever they have a common sucessor in the spectrum. The quotient space of $S$ through this equivalence is called the inductive limit of the spectrum, and denoted by $\lim _{\rightarrow} X_{\alpha}$. On this space we shall consider the quotient topology.

A particular case appears if $X_{\alpha} \subset X_{\beta}$ whenever $\alpha \leqslant \beta$, and the connecting maps are the inclusions. In that case, the inductive limit is said to be the inclusion inductive limit, denoted $\lim _{C} X_{\alpha}$.

If we have a family of continuous maps $\left\{f_{\alpha}: X_{\alpha} \rightarrow Y ; \alpha \in D\right\}$ from the elements of an inductive spectrum $\left\{X_{\alpha}, \zeta_{\alpha, \beta}\right\}$, with values on a topological space $Y$, such that they are compatible $\left(f_{\alpha}=f_{\beta} \circ \zeta_{\alpha, \beta}\right)$, then, by the universal property of inductive limits [8, pp.421-422] there exists a continuous map $f: \lim _{\rightarrow} X_{\alpha} \rightarrow Y$ such that $f_{\left.\right|_{X_{\alpha}}}=$ 
$f_{\alpha}(\alpha \in D)$.

Our first result is concerned with inclusion inductive limits:

THEOREM 2. Let $(D, \leqslant)$ be a directed set containing a countable cofinal subchain. Let $(X, \leqslant)$ be a preordered set such that $X=\lim _{C} X_{\alpha}$, where $\left\{X_{\alpha} ; \alpha \in D\right\}$ is a family of compact preordered spaces (with the induced preorder) such that $X_{\alpha} \subset X_{\beta}$ whenever $\alpha \leqslant \beta$, and each $X_{\alpha}$ being order-separable. Then $\leqslant$ is continuously representable.

Proof: Let $E=\left\{a_{n}\right\}_{n \in \mathbf{N}}$ be a countable cofinal subchain of the directed set $(D, \leqslant)$. The inclusion inductive $\operatorname{limit} \lim _{C} X_{a_{n}}$ relative to the cofinal subchain is homeomorphic to the inductive $\operatorname{limit}_{\mathrm{C}} \lim _{\mathrm{C}} X_{\alpha}$ relative to the whole inductive spectrum [8, Theorem I.7 on p.424]. Let $a_{n}, a_{m} \in E, a_{n} \leqslant a_{m}$, (and then $X_{a_{n}} \subset X_{a_{m}}$ ). Since $\left(X_{a_{n}}, \leqslant \mid X_{a_{n}}\right)$ is a compact preordered set, the quotient topological space $X_{a_{n}} / \sim$ is normally preordered [22, Chapter I, Section 3, Theorem 5]. This assures the existence of a continuous utility function $g_{a_{n}}$ that represents $X_{a_{n}} / \sim[21$, Theorem 1]. Thus, composing with the projection map, there exists a continuous utility function $f_{a_{n}}$ representing $\left(X_{a_{n}}, \leqslant \mid X_{a_{n}}\right)$.

Now, making a slight modification of Theorem 4.2 in [13], it follows that the utility function $f_{a_{n}}$ admits a continuous utility extension $f_{a_{m}}$ to $\left(X_{a_{m}}, \leqslant \mid X_{a_{m}}\right)$, (that is: $f_{a_{n}}=f_{a_{m} \mid X_{a_{n}}}$, and $f_{a_{m}}$ is a utility representation of $\left.\left(X_{a_{m}}, \leqslant \mid X_{a_{m}}\right)\right)$.

We can define a global family of maps $\left\{f_{a_{n}}\right\}_{n \in \mathbb{N}}$ having the above property (that is: $f_{a_{n}}=f_{a_{m} \mid X_{a_{n}}}$ if $\left.X_{a_{n}} \subset X_{a_{m}}\right)$. To get this family, we proceed as follows. Construct $f_{a_{1}}$. If $a_{1} \leqslant a_{2}$ let $f_{a_{2}}$ be an extension of $f_{a_{1}}$ to $X_{a_{2}}$. If, on the contrary, $a_{2} \leqslant a_{1}$, let $f_{a_{2}}$ be the restriction of $f_{a_{1}}$ to $X_{a_{1}}$. In general, suppose $f_{a_{k}}$ constructed and let us define $f_{a_{k+1}}$. If $a_{k} \leqslant a_{k+1}$ let $f_{a_{k+1}}$ be an extension of $f_{a_{k}}$ to $X_{a_{k+1}}$. Otherwise, let $a_{h} \in\left\{a_{1}, \ldots, a_{k}\right\}$ be such that $a_{k+1} \leqslant a_{h}, a_{h}$ being the smallest with that property, and let $f_{a_{k+1}}$ be the restriction of $f_{a_{h}}$ to $X_{a_{k+1}}$.

Once the family $\left\{f_{a_{n}}\right\}_{n \in \mathbf{N}}$ has been constructed, the universal property of inductive limits guarantees the existence of a continuous function $f: \lim _{\subset} X_{a_{n}} \rightarrow \mathbb{R}$ such that $f_{\mid X_{a_{n}}}=f_{a_{n}}$, for every $a_{n} \in E$. It is plain that $f$ is a continuous utility function that represents $(X, \leqslant)$.

The above result can be applied to locally compact $\sigma$-compact preordered spaces $[8, \mathrm{p} .240]$ whose preorder is order-separable on compact subsets. This is a wide class of preordered spaces, which includes the most commonly used in General Equilibrium Theory. (See [25] or [18]).

Proposition 3. Let $(X, \tau)$ be a locally compact $\sigma$-compact Hausdorff space equipped with a closed preorder $\leqslant$. Suppose that the preorder is order-separable on compact subsets. Then there exists a continuous utility function on $X$. 
Proof: In view of Theorem 2, it is enough to see that $(X, \tau)$ is the inclusion inductive limit of an increasing sequence of compact subsets. If $X$ is compact, the result is obvious. Otherwise, since $X$ is $\sigma$-compact, there exists an increasing family of compact subsets $\left(K_{n}\right)_{n \in \mathbb{N}}, K_{n} \subset K_{n+1}$ such that $X=\bigcup\left(K_{n}\right)$. Let $x_{n} \in K_{n+1}-K_{n}$.

Now, because $X$ is Hausdorff, there exists an open subset $O_{n}$ such that $K_{n} \subset O_{n}$ and $x_{n} \notin O_{n}\left[24\right.$, Theorem II 2.5]. Moreover, there exists an open subset $U_{n}$, with compact closure $\mathrm{Cl} U_{n}$ such that $K_{n} \subset U_{n} \subset \mathrm{Cl} U_{n} \subset O_{n}$ [24, Theorem II 2.7]. Consider now the increasing family of compact subsets $\left(\mathrm{Cl} U_{n}\right)_{n \in \mathbb{N}}$. We have that $X=\bigcup\left(\mathrm{Cl} U_{n}\right)$.

Thus, in order to show that $X$ is the inclusion limit of that family, it is enough to prove that the final topology on $X$ coincides with $\tau$. A subset $Y \subset X$ is open in the final topology if, by definition, its intersections with the elements $\mathrm{Cl} U_{n}$ are all open (in the relative topologies of $\mathrm{Cl} U_{n}$ ). Therefore, it is plain that a $\tau$-open subset $Y$ is also open in the final topology. Conversely, if a subset $Y$ is such that all the intersections with the elements $\mathrm{Cl} U_{n}$ are relative-open, it follows that for every $n \in \mathbb{N}$ there exists a $\tau$-open subset $Y_{n}$ such that $Y_{n} \cap \mathrm{Cl} U_{n}=Y \cap \mathrm{Cl} U_{n}$. Then $Y_{n} \cap \mathrm{Cl} U_{n} \cap U_{n}=Y \cap \mathrm{Cl} U_{n} \cap U_{n}=Y \cap U_{n}$, and $Y \cap U_{n}$ is $\tau$-open. Thus $Y=\bigcup\left(Y \cap U_{n}\right)$ is $\tau$-open.

\section{REMARKS.}

(i) It is remarkable that no additional condition (as, for example, "spaciousness", see [23]) is imposed on the preorder $\leqslant$ to guarantee its continuous representability.

(ii) In particular, the preorder $\leqslant$ may or may not be complete. This is important, because most of the results on the representability of preorders that appear in the economics literature refer to complete preorders.

(iii) Proposition 3 generalises a result by Levin stated under the additional assumptions of separability and metrisability of $X$. (See [16] and [17]).

The hypotheses of Theorem 2 and Proposition 3 demand the preorder to be orderseparable. In the next theorem we use inductive limits to get general results of representability of broader classes of spaces than those stated in Theorem 2 .

THEOREM 4. Let $(\leqslant n)_{n \in \mathbb{N}}$ be a family of closed preorders on a topological space $X$. Let $D=\left\{\alpha_{n, m}\right\}_{n, m \in \mathbb{N}}$ be a set of indexes such that, for every $n \in \mathbb{N}$, the family $\left\{X_{\alpha_{n, m}}\right\}_{m \in \mathbb{N}}$ is an inductive spectrum satisfying:

(i) each $X_{\alpha_{n, m}}$ is an order-separable compact preordered space with respect to the preorder $\leqslant n$.

(ii) $X=\lim _{\subset} X_{\alpha_{n, m}}(n$ fixed, $m \in \mathbb{N})$.

Let $\leqslant$ be a preorder on $X$ such that: 
(a) $\leqslant X_{\alpha_{n, m}} \subset \leqslant n$, for every index $\alpha_{n, m} \in D$ (that is: if $x, y \in X_{\alpha_{n, m}}$ then $x \leqslant y($ in $X)$ implies $x \leqslant_{n} y$ (in $\left.X_{\alpha_{n, m}}\right)$ ).

(b) given $x, y \in X, x<y$, there exists $\alpha_{n_{0}, m_{0}} \in D$ such that $x<{ }_{n_{0}} y$.

Then $(X, \leqslant)$ is representable by a continuous utility function.

Proof: It follows, from conditions (i) and (ii) and Theorem 2, that for every $n \in \mathbb{N}$ there exists a continuous function $u_{n}: X \rightarrow \mathbb{R}$, such that $u_{n}$ is a utility function with respect to $\leqslant_{n}$. Notice that hypotheses (ii) and (a) show that $u_{n}$ is an increasing function with respect to $\leqslant$.

We can also assume, without loss of generality, that $u_{n}(x) \in[0,1]$. Now consider the function $u(x)=\sum_{n \in \mathbb{N}} u_{n}(x) / 2^{n}$. for $\leqslant$.

It is easy to check, using condition (b), that $u(x)$ is a continuous utility function

We illustrate with an example the previous theorem.

EXAMPLE. Let $X=(0,1)^{\mathbf{N}}$, equipped with the product topology and the usual (coordinatewise) preorder $\leqslant$.

For every $m \in \mathbb{N}$, set $X_{m}=\left\{\mathrm{x}=\left(x_{1}, x_{2}, \ldots, x_{i}, \ldots\right) \in(0,1)^{\mathbb{N}} ; 1 / m \leqslant x_{i} \leqslant\right.$ $(m-1) / m(i \in \mathbb{N})\}=[1 / m,(m-1) / m]^{\mathbf{N}}$.

On $X$ we shall consider the following family of closed preorders $\left\{\leqslant_{n} ; n \in \mathbb{N}\right\}$. The preorder $\leqslant_{n}$ is defined by:

$$
\mathbf{x} \leqslant n \mathbf{y} \quad \text { if and only if } \quad x_{n} \leqslant y_{n} .
$$

By Tychonoff's theorem, each $X_{m}$ is compact.

Moreover, it is clear that each $\left(X_{m}, \leqslant_{n}\right)$ is an order-separable compact preordered space and $X$ is the inclusion inductive limit of the family $\left\{\left(X_{m}, \leqslant_{n}\right)\right\}$.

Plainly $\leqslant \mid x_{m} \subset \leqslant_{n}$, and clearly, the other hypotheses of the statement of Theorem 4 hold in this case.

This inductive limit is not compact. Neither it is order-separable (to convince you, observe that the order-dense sets have the same cardinality as $\mathbb{Q}^{N}$, which is not countable).

Nevertheless, By Theorem 4, $X$ is representable by a continuous utility function. For instance, a possible representation could be the following:

$$
\mathbf{x}=\left(x_{1}, x_{2}, \ldots, x_{i}, \ldots\right) \in X \rightarrow u(\mathbf{x})=\sum_{i \in \mathbb{N}}\left(x_{i} / 2^{i}\right) \in \mathbb{R} .
$$

We conclude this section by giving an application of Theorem 2 in the context of infinitedimensional topological vector spaces. 
Theorem 5. Let $E$ be a separable topological vector space and $\leqslant$ a weak* continuous complete preorder on $E^{\prime}$. Then there exists a utility function on $E^{\prime}$ which is continuous with respect to the strong topology.

Proof: First of all because $\leqslant$ is a complete preorder, it follows that $\leqslant$ is closed (see $[1$, p.4]). Let $V \subset E$ be a neighbourhood of the origin 0 and $B(n)=\{f \in$ $E^{\prime} ;|f(x)| \leqslant n$, for every $\left.x \in V\right\} \quad(n \in \mathbb{N})$. By the Banach-Alaoglu theorem [26, p.202] each $B(n)$ is a weak*-compact subset of $E^{\prime}$ and, since $E$ is separable, it is well-known that $B(n)$ endowed with the weak* topology is metrisable (see [26]). Therefore (see $[8, \mathrm{p} .233]) B(n)$ is a weak*-separable topological space.

Now consider the preorder $\leqslant$ restricted to $B(n)$. It follows by a classical result due to Eilenberg (see [9]) that $B(n)$ is an order-separable topological preordered space. Define $B^{\prime}(n)=\left\{f \in E^{\prime} ;|f(x)|<n\right.$, for every $\left.x \in V\right\}$. Because each $B^{\prime}(n)$ is an open set in the strong topology, it is follows that $E^{\prime}=\lim _{\subset} B^{\prime}(n)$ (in the strong topology). Thus, by Theorem 2 , there is a strongly continuous utility function on $E^{\prime}$.

REMARK. By following the proof of Theorem 5 we could derive a weak*-continuous utility function since, under the hypotheses of Theorem $5, E^{\prime}$ is order-separable. Indeed, the quotient space $E^{\prime} / \sim$ is an order-separable total order and it is well-known (see [15]) that $E^{\prime} / \sim$ is representable by a utility function continuous in the order topology. Finally it is enough to compose with the projection map $p: E^{\prime} \rightarrow E^{\prime} / \sim$ to get the continuous utility function. Notice however, that $E^{\prime} \neq \lim _{\subset} B(n)$ in the weak* topology.

\section{Applications}

In this section we present two notable applications to economics of the previous concepts and results. The first one deals with the idea of "local behaviour" and "local utility". The second one is an alternative proof and generalisation of the Arrow-Hahn theorem on the existence of continuous utility functions. (See [2, pp.82-87].)

4.1 LOCAL UTILITY. Let $\leqslant$ be a preference relation on a topological space (or differentiable manifold) $X$ and $C$ a class of real-valued continuous (or differentiable) utility functions on $X$. In certain situations, one is only interested in the local behaviour of the functions in the class $C$. For example, let us suppose that a consumer is currently consuming a commodity bundle $x \in X=\mathbb{R}^{n}$. Then the consumer may only be interested in comparing the bundle $x$ with small variations in $x$.

One reason for such behaviour is that "large changes" are feared and distrusted because habitual behaviour is psychologically non-threatening.

Alternatively, if one is considering differentiable utility functions and is interested in applying a theorem such as the implicit function theorem (to compute, for example, 
the slope of an indifference curve at a point), then only small variations are permissible. Under these circumstances, it is natural to identify utility functions that agree on a neighbourhood of the point $x$.

These ideas may be made formal and precise in the following way:

Definition: Let $X$ be a topological space. A presheaf $F$ of sets on $X$ is given by the following conditions (see [27]):

(1) for each open set $U \subset X$, there exists a set $F(U)$ called the set of sections over $U$;

(2) for each pair of open sets $V \subset U$ of $X$, there exists a restriction map $\varphi_{V}^{U}: F(U) \rightarrow F(V)$ such that

(a) for all open $U, \varphi^{U} U=i d_{U}$,

(b) whenever $W \subset V \subset U$ are open sets of $X$, then $\varphi^{U} W=\varphi^{V}{ }_{W}$ o $\varphi^{U} V$.

Now let $(X, \leqslant)$ be a preordered topological space. The presheaf $C$ of realvalued continuous utility functions on $X$ is defined by declaring that $C(U)$ is the set of continuous utility functions $U \rightarrow \mathbb{R}$ for $U$ open in $X$, and the restriction map $\varphi^{U}{ }_{V}: C(U) \rightarrow C(V), V \subset U$ is given by the restriction of the function $f \in C(U)$ to the subset $V$. It is readily verified that this does define a presheaf on $X$.

Let now $x$ be an arbitrary but fixed point in $X$ and $C$ the presheaf defined above. Consider the set $D$ of the open subsets of $X$ such that $x \in U$. Define a relation $\leqslant$ on $D$ by declaring $U \leqslant V$ if and only if $V \subset U$. Then $(D, \leqslant)$ is a directed set.

Now associate with any open set $U$ of $X$ such that $x \in U$ the set $C(U)$ of realvalued continuous utility functions on $U$ and define the connecting maps $\varphi_{U, V}, V \subset U$, to be the restrictions of the functions in $C(U)$ to the subset $V$. Then clearly, we have an inductive spectrum of spaces and connecting maps.

Now the inductive (or direct) limit of this spectrum is called the stalk $C_{x}$ of the presheaf $C$. The members of this stalk $C_{x}$ are called germs (of sections of $C$ ).

Consider now the members of the stalk $C_{x}$. Two members $s_{x}$ and $t_{x}$ with $s_{x} \in$ $C(U)$ and $t_{x} \in C(V)$ of the stalk of this presheaf are equal if and only if they have a common sucessor in the spectrum. Therefore, $s_{x}=t_{x}$ if and only if there exists an open set $W \subset U \cap V$ such that the functions $s$ and $t$ agree on $W$.

Therefore, two functions in the stalk $C_{x}$ are identified if they agree on some neighbourhood of $x$. (See also [8, Example 3 on p.422]).

4.2 The Arrow-Hahn theorem revisited. We now give the following important application of the concept of an inductive spectrum of spaces. First, we need some notation.

Notation: Let $\leqslant$ be a preorder on a set $X$. We denote the upper section $\{y \in$ 
$X ; x \leqslant y\}$ of $x$ by $C(x)$.

DEFINITION: Let $F$ be a subset of a normed linear space $E$. If for each $x \in E$ there is $z \in F$ such that $d(x, z)=d(x, F)$, where $d(x, y)=\|x-y\|$, then $F$ is said to be proximinal in $E$.

Definition: Let $\leqslant$ be a preorder on a subset $X$ of a Banach space $E$. We say that $\leqslant$ is locally non-satiated if for each $x \in X$ and each neighbourhood $U$ of $x$ there is $y \in U$ such that $x<y$.

We now prove the following theorem on the existence of a continuous utility function on a subset of a Banach space $E$. This generalises the Arrow-Hahn theorem (see [2, pp.82-87]) to an infinite dimensional space.

Theorem 6. Let $E$ be a Banach space with predual $F$ and $\leqslant$ a weak*continuous complete preorder on a weak ${ }^{*}$-closed convex subset $X$ of $E$. Suppose that $\leqslant$ is locally non-satiated with respect to the weak*-topology. Then there is a continuous utility function on $X$.

Proof: Choose a point $x_{0} \in X$. We prove first that there is a continuous utility function on $C\left(x_{0}\right)$. Define a function $U$ on $C\left(x_{0}\right)$ by $U(x)=\inf \left\{\left\|x_{0}-y\right\| ; y \in C(x)\right\}$. Since $\leqslant$ is weak*-continuous, the set $C(x)$ is weak*-closed. Now each weak*-closed subset of a Banach space with a predual is proximinal $[14$, p.116]). Therefore, the function $U$ is well-defined. As in [2, pp.82-87], we may prove that $U$ is a continuous utility function. The assumption of local non-satiation of $\leqslant$ with respect to the weak*topology is needed to prove the upper semicontinuity of $U$.

We claim next that if there is a point $z \in X \backslash C\left(x_{0}\right)$ then there exists a continuous utility function $V$ on $C(z)$ such that $V(x)=U(x)$ for every $x \in C\left(x_{0}\right)$, that is $V$ is a continuous extension of $U$. To that end, observe first that $U\left(x_{0}\right)=0$.

Now define a function $\boldsymbol{H}: C(z) \rightarrow \mathbb{R}$ by $\boldsymbol{H}(\boldsymbol{x})=\inf \{\|z-y\| ; y \in C(x)\}$. By arguing as above, we see that $H$ is a continuous utility function. Observe that $x_{0} \in C(z)$ since $z \in X \backslash C\left(x_{0}\right)$. Therefore $H\left(x_{0}\right)=d$ for some real number $d$.

Next, notice that $H$ maps the order-interval $\left[z, x_{0}\right]$ into $[0, d]$, where $H(z)=0$ and $H\left(x_{0}\right)=d$. Since there exists a strictly increasing homeomorphism $h$ mapping $[0, d]$ into $[-1,0]$ we may assume without loss of generality that $H$ is a continuous utility function on $C(z)$ with values in $[-1, a]$, with $d<a$, such that $B$ maps the order-interval $\left[z, x_{0}\right]$ into $[-1,0], H(z)=-1$ and $H\left(x_{0}\right)=0$.

Now define a real-valued function $V$ on $C(z)$ by $V(x)=U(x)$ if $x_{0} \leqslant x$ and $V(x)=H(x)$ if $z \leqslant x \leqslant x_{0}$. Clearly, $V$ is a utility function on $C(z)$. It is continuous by the Glueing Lemma [6, p.151]. Hence $V$ is a continuous extension of $U$ and the claim is proved.

It should be noted that both $U$ and $V$ are "metric utility functions" in the sense 
that they are defined in terms of the canonical metric of $E$. Observe, however, that if $x_{0}<a$ then $V(a)$ may not be equal to $H(a)$.

If $E$ has a $\leqslant$-smallest element then we may let $x_{0}$ be that $\leqslant$-smallest element and the theorem is proved.

So suppose that $E$ has no $\leqslant-$ smallest element. Let $B(0, n)$ for $n=1,2, \ldots$ be the closed ball about the origin of radius $n$.

Each $B(0, n)$ is weak*-compact by the Banach-Alaogu theorem [26, p.202].

Therefore $B(0, n) \cap X$ is a weak*-compact subset of $X$ for each positive integer $n$. This implies that there is a $\leqslant$-smallest element in $B(0, n)$ for each $n$ because $\leqslant$ is weak*-continuous. Now let $n_{1}$ be the least positive integer such that $B\left(0, n_{1}\right) \cap X$ is nonempty, and let $x_{1}$ be the $\leqslant$-smallest element of $B\left(0, n_{1}\right) \cap X$. It follows from the hypothesis that $X$ has no $\leqslant$-smallest element that there exists a positive integer $j$ such that $z \in B\left(0, n_{j}\right) \cap X$ and $z<x_{1}$. Let $n_{2}$ be the least such integer that is greater than $n_{1}$ and let $x_{2}$ be the $\leqslant$-smallest element of $B\left(0, n_{2}\right) \cap X$.

By continuing this argument, we see that there exists a subsequence $\left(x_{n_{i}}\right)$ of $\left(x_{n}\right)$ such that $B\left(0, n_{i}\right) \cap X$ is not empty and $B\left(0, n_{1}\right) \subset B\left(0, n_{2}\right) \subset \ldots$

Let $S=\left\{n_{1}, n_{2}, \ldots\right\}$. Now for each $n_{i} \in S$ there exists by what has been proved before a continuous function $U_{n_{i}}$ defined on $C\left(x_{n_{i}}\right)$. If $n_{i}<n_{j}$ the function $U_{n_{j}}$ is a continuous extension of the function $U_{n_{i}}$.

Therefore the family $C\left(x_{n_{1}}\right) \subset C\left(x_{n_{2}}\right) \subset \ldots$ is a nested family of spaces. Let the connecting maps $f_{n_{i}, n_{j}}, n_{i}<n_{j}$, be the inclusion maps. We therefore have an inductive spectrum of spaces and connecting maps. Observe that $X$ is the inductive limit of these spaces.

By the universal property of inductive limits we may conclude as before that there exists a continuous utility function $U$ on the inductive limit $X$ of the spaces $C\left(x_{n_{i}}\right)$ such that the restriction of the function $U$ to $C\left(x_{n_{i}}\right)$ is just $U_{n_{i}}$. The function $U$ is the required utility function on $X$.

\section{RemaRKs.}

(1) Since $\mathbb{R}^{n}$ has a predual and the Euclidean topology is the weak*-topology, it follows that Theorem 6 proved above is a generalisation of the finitedimensional Arrow-Hahn theorem to infinite-dimensional spaces with preduals.

In addition, observe that since the extension procedure used in Theorem 6 above is different from the Arrow-Hahn procedure we have also given a new proof of this important theorem.

(2) An alternative extension procedure that may be used to prove the ArrowHahn theorem is based on Nachbin's extension theorem (see [22] and 
[19]).

(3) It is an open question whether Theorem 6 proved can be extended to Banach spaces which do not have preduals.

(4) Inductive limits lead to another striking application to economics, in the construction of infinite models of General Competitive Equilibrium, in particular in the setting of the Overlapping Generations Model. (See [1, p.240 and following].)

\section{REFERENCES}

[1] C.D. Aliprantis, D.J. Brown and O. Burkinshaw, Existence and optimality of competitive equilibria (Springer-Verlag, Berlin, Heidelberg, New York, 1990).

[2] K.J. Arrow and F.H. Hahn, General competitive analysis (Holden Day, San Francisco, 1971).

[3] R.J. Aumann, 'Subjective programming', in Human Judgements and Optimality, (M.W. Shelly, II and G.L. Bryan, Editors) (John Wiley and Sons, New York, 1964).

[4] J.C. Candeal and E. Induráin, 'Sobre caracterizaciones topológicas de la representabilidad de cadenas mediante funciones de utilidad', Revista Española de Economía 7 (2) (1990), 235-244.

[5] J.C. Candeal and E. Induráin, 'Representación numérica de órdenes totales', Rev. Real Acad. Cienc. Exact. Fis. Natur. Madrid 84 (1990), 415-428.

[6] F. Croom, Principles of topology (Saunders College Publishing, Philadelphia, 1989).

[7] G. Debreu, 'Representation of a preference ordering by a numerical function', in Decision processes, (R. Thrall et al., Editors) (John Wiley and Sons, New York, 1954), pp. 159-166.

[8] J. Dugundji, Topology (Allyn and Bacon, Boston, 1966).

[9] S. Eilenberg, 'Ordered topological spaces', Amer. J. Math. 63 (1941), 39-45.

[10] I. Fleischer, 'Numerical representation of utility', J. Soc. Indust. Appl. Math. 9 (1961), 48-50.

[11] G. Herden, 'On the existence of utility functions', Math. Social Sci. 17 (1989), 297-313.

[12] G. Herden, 'On the existence of utility functions II', Math. Social Sci. 18 (1989), 107-117.

[13] G. Herden, 'Some lifting theorems for continuous utility functions', Math. Social Sci. 18 (1989), 119-134.

[14] R. Holmes, Geometric functional analysis and its applications (Springer-Verlag, Berlin, Heidelberg, New York, 1975).

[15] J.Y. Jaffray, 'Existence of a continuous utility function: an elementary proof', Econometrica 43 (1975), 981-983.

[16] V.L. Levin, 'Measurable utility theorems for closed and lexicographic preference relations', Soviet Math. Dokl. 27 (3) (1983), 639-643.

[17] V.L. Levin, 'General Monge-Kantorovich problem and its applications in measure theory and mathematical economics', in Functional Analysis, Optimization and Mathematical Economics, (Lev J. Leifman, Editor) (Oxford University Press, 1990). 
[18] A Mas-Colell, 'An equilibrium existence theorem without complete or transitive preferences', J. Math. Econom. 1 (1974), 237-246.

[19] G.B. Mehta, 'A new extension procedure for the Arrow-Hahn theorem', Internat. Econom. Rev. 22 (1981), 113-118.

[20] G.B. Mehta, 'On a theorem of Fleischer', J. Austral. Math. Soc. Ser. A 40 (1986), 261-266.

[21] G.B. Mehta, 'Existence of an order-preserving function on normally preordered spaces', Bull. Austral. Math. Soc. 34 (1986), 141-147.

[22] L. Nachbin, Topology and order (Van Nostrand, Princeton, N.J., 1965).

[23] B. Peleg, 'Utility functions for partially ordered topological spaces', Econometrica 38 (1970), 93-96.

[24] W. Rudin, Real and complex analysis (McGraw-Hill, New York, 1966).

[25] D. Schmeidler, 'Competitive equilibria in markets with a continuum of traders and incomplete preferences', Econometrica 37 (1969), 568-585.

[26] C. Swartz, Introduction to functional analysis (Marcel Dekker, New York, 1992).

[27] B.R. Tennison, Sheaf theory (Cambridge University Press, Cambridge, U.K., 1975).

Departamento de Análisis Económico

Universidad de Zaragoza

Facultad de Ciencias Económicas y Empresariales

Gran Vía 2-4

50005-Zaragoza

Spain

Department of Economics

The University of Queensland

St. Lucia

Queensland

Australia 4067
Departamento de Matemática e Informática

Universidad Pública de Navarra

Campus Arrosadía s.n. 31006-Pamplona

Spain 